\title{
Study of the Effects of Edge Morphology on Detector Performance by Leakage Current and Cathodoluminescence
}

\author{
J. Crocco, H. Bensalah, Q. Zheng, F. Dierre, P. Hidalgo, J. Carrascal, O. Vela, J. Piqueras, and E. Diéguez
}

\begin{abstract}
The Vertical Gradient Freeze (VGF) method has been used to grow high resistivity Cadmium Zinc Telluride (CZT) for high energy radiation applications. In this work, the effect of lapping and polishing the lateral edges of planar detectors is studied. Expectations that improved surface morphology of the edges should correlate with reduced surface leakage current are shown to be erroneous. The effect of various types of lateral edge treatments on detector performance was observed before and after each surface modification. Complementary results were obtained using I-V, Cathodoluminescence (CL), and gamma ray response measurements using 133Ba. As a result, a quick and easy method is reported which minimizes leakage current and actually enhances detector performance through the introduction of surface defects. It is demonstrated that the introduction of radiative recombination centers helps reduce surface leakage current in the detector by a factor of up to $200 \%$, depending on the surface treatment. The purpose of this work is to identify material processing steps for fabricating planar devices based on CZT for gamma ray spectroscopy.
\end{abstract}

Index Terms-Cadmium zinc telluride (CZT), gamma ray detection, leakage current, surface morphology, vertical gradient freeze (VGF).

\section{INTRODUCTION}

$\mathbf{T}$ HE advantages of Cadmium Zinc Telluride (CZT) based detectors are their wide bandgap, high atomic number, and room temperature operability. CZT-based detectors provide higher energy resolution compared with scintillator detectors (which behave as indirect convertors and require a second stage, such as photomultiplier tubes, to convert low energy scintillation photons into electronic charge, decreasing the signal to noise ratio), while commercially available semiconductors such as Ge require cryogenic working temperatures (and equipment) due to the high leakage currents associated with a low energy band gap $(0.67 \mathrm{eV}$ at $300 \mathrm{~K}$ respectively).

Manuscript received November 13, 2010; revised March 15, 2011 and May 09, 2011; accepted May 10, 2011. Date of publication June 27, 2011; date of current version August 17, 2011. This work was supported by the COCAE FP7 Project (http://www.cocae.eu)

J. Crocco, H. Bensalah, Q. Zheng, F. Dierre, and E. Diéguez are with the Crystal Growth Laboratory (CGL), Materials Physics Department, Universidad Autónoma de Madrid, 28049 Madrid, Spain (e-mail: jerome.crocco@gmail.com).

P. Hidalgo and J. Piqueras are with the Departamento de Física de Materiales, Facultad de Ciencias Físicas, Universidad Complutense de Madrid, 28040 Madrid, Spain.

J. Carrascal and O. Vela are with the CIEMAT, E-28040 Madrid, Spain.

Color versions of one or more of the figures in this paper are available online at http://ieeexplore.ieee.org.

Digital Object Identifier 10.1109/TNS.2011.2157703

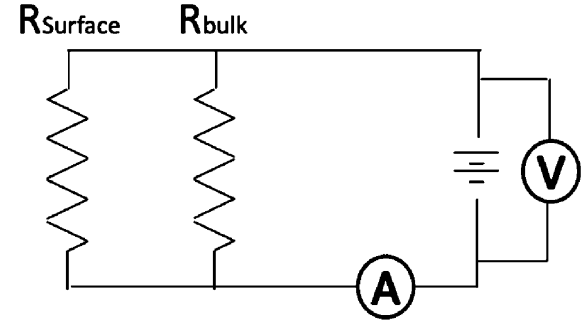

Fig. 1. Simplified circuit diagram of a CZT detector whose surface leakage current is limited by the surface resistivity.

There are several challenges to producing detector grade material based on CZT, one of which includes the mechanical engineering processes such as ingot slicing, wafer lapping and polishing. H. Yoon et al. describes how the effects of saw-cutting, lapping, polishing, and etching effect crystalline periodicity and sub-surface disorder in CZT using High Resolution $\mathrm{X}$-ray Diffraction [1]. This sub-surface disorder is a result of the mechanical deformation of the surface and destruction of lattice periodicity.

For this reason, the type treatment given to the lateral sides of a functioning detector is paramount. Not only does the deformation of the CZT surface by slicing and lapping impart dislocations into the material, but the extended depth of these dislocations could provide a larger volume of avenues for the surface leakage current to pass between anode and cathode and effectively degrade the signal to noise ratio of the detector. The lateral edges of the detector can act as a 'short circuit' under the correct circumstances, as shown below in Fig. 1. For this reason, special consideration to the treatment to the lateral sides is required, in order to identify practical methods for enhancing detector performance.

Mechanical lapping refers to the use of a rigid plate (glass in this work) in conjunction with rolling abrasives to produce flat, yet rough surfaces. Mechanical polishing involves the abrasion of material from the surface by using polishing cloths and abrasive slurries in which the abrasive is fixed (to some degree) within the pores of the polishing pad. The net size of the exposed particle is reduced and the surfaces produced are substantially smoother (and normally reflective).

Several works have been presented using diamond, alumina, or magnesium oxide based slurries for mechanically polishing CZT. Others techniques have also been implemented using chemo-mechanical polishing (CMP) methods with abrasives such as $\mathrm{SiO} 2$ nanoparticles. [2] 
There are several papers which discuss the principles of chemical polishing as well. For example Singh et al. outlines the optimization of several physical parameters for Cd0.96Zn0.04Te used to meet the demands for thin film growth by Molecular Beam Epitaxy [3]. P. Morovec et al. presents the effects of load, slurry concentration, bromine concentration, and pad type on material removal rates [4]. Still other results have demonstrated that by using weaker oxidants based on iodine with lower redox potential value, compared with bromine, the surface claimed to be more stable with time, and with a surface roughness comparable to that obtained with bromine [5]

It has been demonstrated how surface recombination is affected by surface treatment and that higher electron recombination rates can be obtained by using polished only surfaces [6]. While this may be beneficial for the cathode or anode surface preparation, how it may effect the lateral edges is not immediately clear. Other work has also shown that contacts sputtered on 'as-polished' surfaces perform better than sputtered contacts on chemically etched surfaces, leading to lower leakage currents [7].

In this work, a broad range of processes are investigated including lapping, mechanical polishing, and chemical polishing. A key differentiator is that this study illustrates how the surface treatment of the lateral edges of CZT planar detectors affects their I-V characteristics.

\section{EXPERIMENTAL}

\section{A. Crystal Growth}

High resistivity CZT ingots were grown by the VGF method for this study. The VGF system used in this work has 5 independently controlled heater zones used to create a dynamic temperature profile across the ingot. All three ingots were grown using 7 N CZT material loaded into a Pyrolytic Boron Nitride (pBN) crucible, and were all doped with between 3-5 ppm of Indium to achieve electrical compensation.

\section{B. Detector Fabrication}

Wafers were sliced from the ingot using a diamond wire saw, and subsequently lapped and polished using a Logitech PM5 and CP3000 respectively. Detector material with dimensions of $8 \mathrm{~mm} \times 8 \mathrm{~mm} \times 1.5 \mathrm{~mm}$ and $15 \mathrm{~mm} \times 25 \mathrm{~mm} \times 2 \mathrm{~mm}$ were used in this work. Prior to metallization, samples were etched in $2 \%$ Bromine methanol for 30 seconds. Contacts were deposited using the gold electroless method. This chemical method of deposition creates a strong chemical bond between the contact and the semiconductor when compared to physical deposition methods such as sputtering and evaporation.

After electrodes were deposited, the lateral surfaces of the detector were subsequently lapped and polished, as can be seen in Fig. 2. The effect of each subsequent surface processing step on leakage current and gamma response is presented in this work, maintaining the same original cathode/anodes throughout.

The abrasives used in this work for lapping and polishing the edges of the detectors include 9 and $3 \mu \mathrm{m} \mathrm{Al}_{2} \mathrm{O}_{3}$ distributed

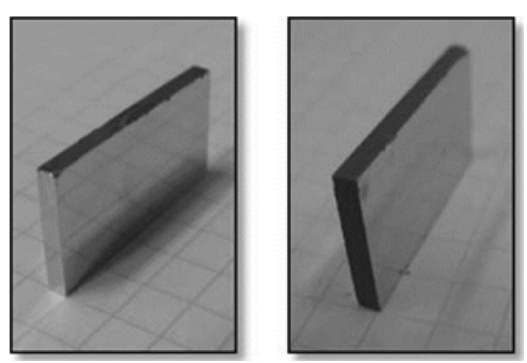

Fig. 2. $15 \mathrm{~mm} \times 25 \mathrm{~mm} \times 2 \mathrm{~mm}$ CZT samples used in this study after (left) gold electroless process and after lateral edge lapping (Right). $8 \mathrm{~mm} \times 8$ $\mathrm{mm} \times 15$ CZT samlples were also used.

by Logitech, UK as well as $1 \mu \mathrm{m}$ monocrystaline diamond distributed by Eminess Technologies (U.S.). The chemical polishing solution used in this work is that reported by Singh et al. consisting of $\mathrm{Br}_{2}$ in ethylene glycol [3].

Both free and fixed abrasive machining processes were used for lateral edge treatment (lapping and polishing). For the lapping process, the $9 \mu \mathrm{m}$ and $3 \mu \mathrm{m} \mathrm{Al}_{2} \mathrm{O}_{3}$ abrasives were used for lapping the lateral edges of the detector. Hereafter, these processes will be referred to as $L E L-9$ and $L E L-3$ i.e., lateral edge lapping $9 \mu \mathrm{m}$ Alumina.

For the mechanical polishing process, $3 \mu \mathrm{m} \mathrm{Al}_{2} \mathrm{O}_{3}$ and $1 \mu \mathrm{m}$ diamond abrasives were investigated. As already mentioned a $2 \% \mathrm{Br}_{2}$ in ethylene glycol solution was used for the chemical polishing process. Hereafter, these processes will be referred to as $L E P-3, L E P-1$, and $L E P-C P$ respectively.

\section{Characterization}

Current-Voltage characteristics of detectors with planar contacts were measured using a Keithley 2400 sourcemeter. Resistivity was measured for each detector after surface modification. The change in surface area of each detector as a result of the lapping/polishing process was taken into account when calculating current density. As the bulk resistivity of the material remains constant, changes in I-V are attributed to changes in surface leakage current along the lateral edges after each surface treatment.

Bulk resistivity was measured using (1), where $A$ is detector area, $\rho$ is the sample resistance determined from the I-V curve and $\delta$ is the detector thickness.

$$
\Omega=\frac{A \times \rho}{\delta} .
$$

Samples prepared for CL measurements were cut from the same region of the same ingot. In total, five samples were prepared, one using each surface treatment: lapped using $9 \& 3 \mu \mathrm{m}$ $\mathrm{Al}_{2} \mathrm{O}_{3}$ (LEL-9 and $L E L-3$ ), polished using $3 \mu \mathrm{m} \mathrm{Al}_{2} \mathrm{O}_{3}, 1 \mu \mathrm{m}$ diamond, and chemically polished using the formulation proposed by Singh et al. (LEP-3, LEP-1, LEP-CP respectively). Taking into account that each sample comes from the same region of the ingot, changes in the CL signal are attributed to changes imparted by the different treatments given.

Secondary electron images and cathodoluminiscence (CL) measurements of the lapped and polished CZT surfaces were realized using an FEI-INSPECT and an Hitachi2500 Scanning 
Electron Microscope in conjunction with a R5509 Hamamatsu photomultiplier tube used for CL detection. Electrons were accelerated under a $20 \mathrm{kV}$ potential difference. The samples were cooled to a temperature of $90 \mathrm{~K}$ for these measurements and all of the emission spectra were normalized to the maximum peak emission value.

CZT detector performance was characterized using ${ }^{133} \mathrm{Ba}$ sources. The source was placed $3 \mathrm{~mm}$ from the detector cathode. The detector itself was mounted on a custom Teflon fixture with a gold coated mounting plate (anode) and was contacted using a gold cantilever (cathode). The Teflon fixture was placed within a lead test box with each electrode connected to an AMPTEK A250 preamplifier. The pulse from the detector was further amplified by a shaping amplifier with a shaping time of $1.5 \mu \mathrm{s}$. The shaped signal was digitized and captured using an APTEC multichannel analyzer. The APTEC's control software finally records the data in comma separated values format. As the surface modification affects the surface leakage current, detector performance was measured in terms of the electronic noise introduced into the system (lower than $35 \mathrm{keV}$ peak of $\left.{ }^{133} \mathrm{Ba}\right)$.

The cathode and anode of the detector were protected using a thin film of wax while the edges were subjected to each treatment. This prevented the cathode and anode form being damaged. By using this approach, the electrodes deposited on each detector were the same throughout the entire study, with no need to re-contact the detectors (which could introduce a large degree of error into the study of the gamma response)

To identify optimal working conditions, each detector was first biased through a range of voltages to identify the best working voltage specific to each detector. The surface morphology study on detector performance was then carried out at this voltage (though for some surface treatments, it was not possible to reach this working voltage)

\section{RESULTS}

\section{A. Surface Morphology}

The typical surface morphology of each treatment is presented in Fig. 3. The LEL-9 and LEL-3 treatments exhibit very rough and are optically non-reflective surfaces. From the SEM images, the surfaces do appear somewhat similar.

The LEP-3 treatment produces a reflective surface finish, and contains a high density of scratches and substantial roughness. The LEP-1 and LEP-CP treatments both exhibit smoother surface morphology with lower scratch densities and depths.

\section{B. Current Voltage Characteristics}

1) Mechanical Lapping: The lateral edges of each CZT detector were first lapped using $9 \mu \mathrm{m}$ alumina (LEL-9). This was the starting point of the investigation. Using more than 6 detectors from different CZT ingots, the I-V characteristics were measured in order to ensure the material was of high resistivity. The lateral edges of the detector were then subsequently lapped using $3 \mu \mathrm{m}$ alumina (LEL-3).

After both the LEL-9 and LEL-3, the leakage current of each detector higher than compared with the LEP-3 treatment. This is

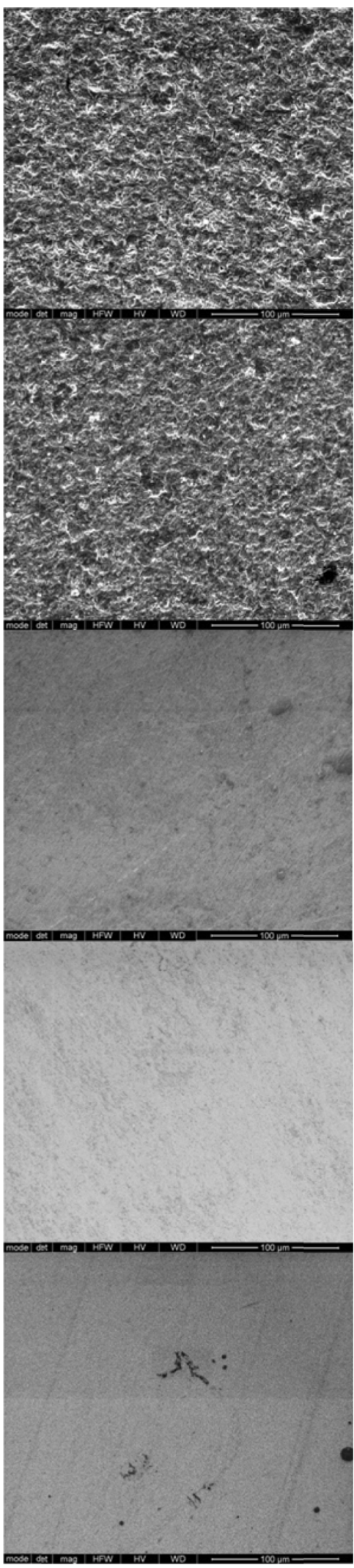

Fig. 3. Lapped and Polished surfaces by SEM. (A) after lapping with $9 \mu \mathrm{m}$ $\mathrm{Al}_{2} \mathrm{O}_{3}$. (B) lapping with $3 \mu \mathrm{m} \mathrm{Al}{ }_{2} \mathrm{O}_{3}$. (C) polishing using $3 \mu \mathrm{m} \mathrm{Al}_{2} \mathrm{O}_{3}$. (D) polishing using $1 \mu \mathrm{m}$ diamond. (E) after chemical polishing.

thought to be related with the surface damage layer imparted by lapping creating dislocations and effectively reducing the electrical resistivity of the surface layer. 


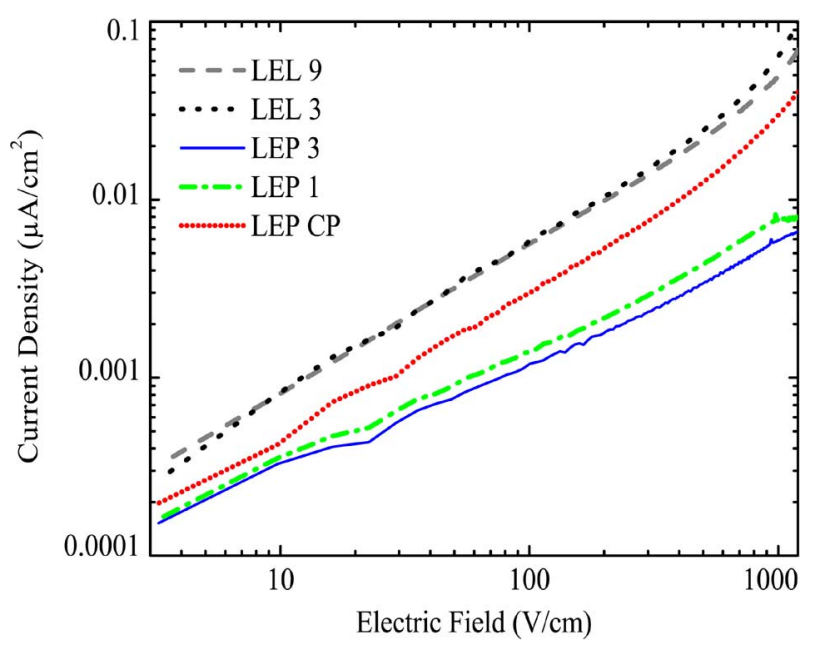

Fig. 4. Typical I-V characteristics of detectors after modifying the lateral edges with different surface treatments.

2) Mechanical Polishing: A reduction in leakage current was obtained after polishing the lateral edges using the LEP-3 treatment. This treatment changes the edges from having a non-reflective matte surface to a mirror-like reflective finish. Leakage current for planar detectors having undergone LEP-3 treatment showed the best $\mathrm{I}-\mathrm{V}$ characteristics of all edge treatments investigated.

After polishing the lateral edges of the detectors using the LEP-1 treatment, the lateral edges of the CZT detector are substantially smoother. The removal rate of this polishing stage is much lower than previous steps, but the surface roughness is substantially reduced from greater than $20 \mathrm{~nm}$ for the LEP-3 treatment to nearly $5 \mathrm{~nm}$ RMS roughness as measured by AFM. Despite this improved surface morphology, detectors having undergone LEP-1 treatment exhibited a slight increase in leakage current, as can be seen in Fig. 4. This work was repeated on more than six different detectors harvested from different ingots to confirm the reproducibility of the result. In each case, there was only a slight increase in leakage current.

3) Chemical Polishing: Finally, the lateral edges of each detector were chemically polished using a the LEP-CP treatment. The cathode and anode of the detectors were protected using a thin wax film, which could be removed afterwards using trichloroethylene. Detectors having undergone LEP-CP treatment exhibited higher leakage current, and showed some instability over a short period of $24 \mathrm{hrs}$. The mechanical lapping and polishing treatments did not exhibit this temporal behavior. A longer term study would be beneficial but did not fall within the scope of this work.

Shown in Fig. 4 are the typical I-V characteristics for one of the detectors used in this study, subjected to all of the treatments. The change in resistivity before and after each subsequent lapping and polishing operation was measured to observe the effects of surface preparation on the performance of a planar detector geometry. The contacts and bulk resistivity have not changed and therefore the change in the I-V characteristics can be attributed to changes in surface leakage current.

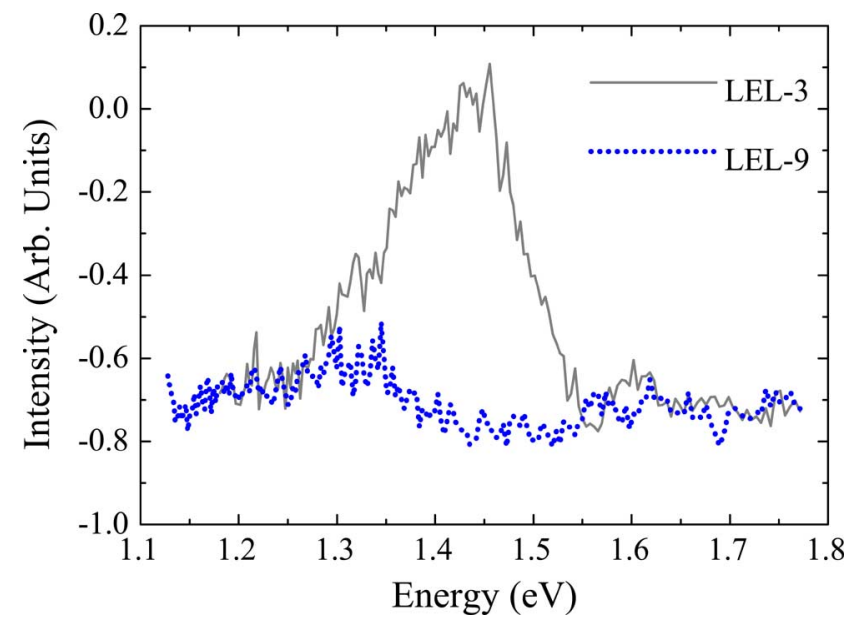

Fig. 5. CL intensity of CZT surface after LEL-9 and LEL-3 treatment.

\section{Cathodoluminescence}

Cathodoluminescence (CL) provides a method to evaluate the dominant radiative trapping centers which exist within the bandgap of CZT, and is sensitive to surface morphology. It provides a method for identifying how different surface treatments can introduce different types of surface trapping levels, by the relative intensity of their radiative emissions. Spectra have been recorded under different excitation conditions of the electron beam on the sample, to account for radiative centers with very different lifetimes. Usually by low excitation, the intensity of the bands associated to defects with low lifetime increase compared to bands associated to defects with a very high lifetime. For CZT, the broad band located at $1.4 \mathrm{eV}$ is strongly related with the A center, but is also associated with impurities in the starting material. More importantly for this work is that this band is also related with surface defects [8]. For this reason, changes in $1.4 \mathrm{eV}$ band and neighboring peaks are presented after each treatment.

1) Cathodoluminescence of Lapped Surfaces: Detectors having undergone $L E L-9$ did not exhibit luminescence under both high and low excitation conditions. The absence of a luminescence peak in the sample lapped using the $9 \mu \mathrm{m} \mathrm{Al}_{2} \mathrm{O}_{3}$ is attributed to non-radiative recombination of electrons being the dominant recombination mechanism.

The CL spectra measured for the sample after $L E L-3$ did in fact exhibit a peak near $1.4 \mathrm{eV}$, but only under low excitation conditions. The presence of this luminescent peak is attributed to a reduction in non-radiative recombination centers as a result of using a smaller abrasive particle which produces less surface damage, and reduces the non-radiative centers. Presented in Fig. 5 are the low excitation CL spectra taken for after each lapping treatment.

2) CL of LEP-3 and LEP-1 Treatment: As the subsurface damage is removed through the mechanical polishing using fine abrasives in conjunction with a porous felt pad, the non-radiative recombination centers are significantly reduced. This is evidenced by the improved signal to noise ratio of the measured CL spectra, as radiative recombination becomes more prominent. 


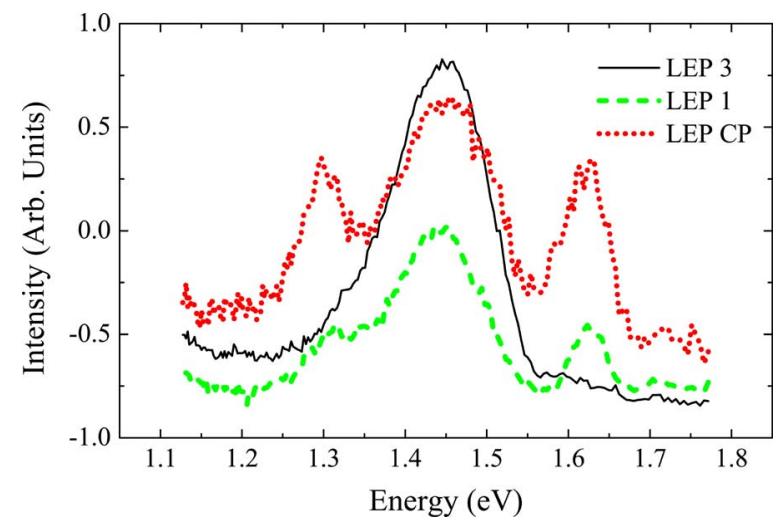

Fig. 6. CL intensity of CZT surface after LEP-3, LEP-1, and LEP-CP treatment.

When the detectors have been subjected to the LEP-3 treatment, a single and broad luminescence band located near $1.4-1.5 \mathrm{eV}$ is observed. It has been observed that after this treatment, the intensity of $1.4 \mathrm{eV}$ band is highest compared with the other situations.

Additional polishing of the detector edges using LEP-1 treatment produces a lower surface roughness and a more uniform surface morphology i.e., finer scratches, low RMS surface roughness and Peak to Valley. The concentration of surface related traps, however are also reduced as three distinct luminescence peaks are identifiable. The presence of these peaks suggests that the concentration of surface related traps at the $1.4 \mathrm{eV}$ peak are being reduced, and other radiative centers related with the bulk (i.e., near band gap emission centered at $1.6 \mathrm{eV}$ and $1.3 \mathrm{eV}$ band related to impurities) can be identified. Presented in Fig. 6 are the CL spectra for the LEP-3, and LEP-1 treatments.

3) CL of LEP-CP Treatment: Chemically polishing produces the lowest surface roughness with low scratch densities and depths. The CL spectrum for the $L E P-C P$ treatment shows very clearly strong peaks located at $1.3 \mathrm{eV}$ and $1.6 \mathrm{eV}$. The ratio of the maximum of the $1.4 \mathrm{eV}$ band to these other peaks has diminished substantially indicating a lower concentration of surface related defects. The chemically polished surface further removes the surface trapping centers imparted by mechanical polishing, revealing the bulk electro-optic properties of the semiconductor.

Presented in Fig. 6 are the CL spectra for CZT LEP-CP treatment. The CL spectra demonstrates the large dependence of the luminescence properties of CZT with the surface preparation. All presented CL spectra are for low excitation conditions.

\section{Gamma Ray Response}

CZT detectors were prepared using the methods described above. The edges of these detectors were first polished using the $L E P-3$ treatment as the starting point for this study, due to the improved I-V characteristics obtained on other detectors. The effects of surface morphology on the detector performance from this point were then studied.

The first observation was that after the $L E L-9$ and $L E L-3$ treatments, detectors could not reach the same operating voltage as was obtained using the $L E P-3$ treatment. Instead, the voltage

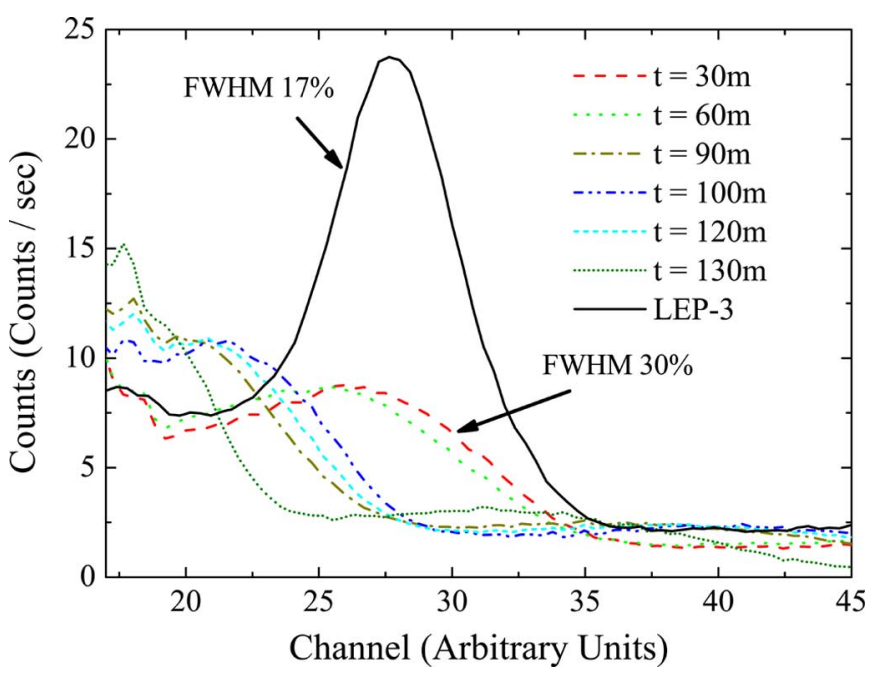

Fig. 7. Detector performance after LEL-9 was poor in terms of FWHM and also changed over the course of 2 hours compared with the same detector after LEP-3 treatment.

had to be reduced by up to $50 \%$ in some cases to obtain a gamma response. In addition, the FWHM of the $35 \mathrm{keV}$ photo peak was larger than after LEP-3 treatment.

Initially, the detector was operative at the reduced bias and gamma spectra could be recorded. However for some detectors, after period of time noise began to dominate the signal and degrade the spectra. In some cases this happened during a single recording measurement, in other cases it happened during a separate trial using the same detector under identical conditions.

Shown below in Fig. 7 is the gamma response of one of the detectors measured during a 2 hour period, after the LEL-9 treatment. The data collection was reset every thirty minutes to collect new data and observe how the detector changes with time. Also presented in this figure is the gamma response of the same detector after LEP-3 treatment.

After the $L E P-1$ and $L E P-C P$ treatment, using the $\mathrm{Br}_{2}$ ethylene glycol solution, it has been demonstrated through $I-V$ measurements that the detector leakage current increases as a result of these surface modifications. The effects which these edge treatments have on detector performance are presented here. Shown below in Fig. 8 is the gamma ray response for a CZT planar detector measured after the $L E P-3$ treatment and after subsequent $L E P-1$ and $L E P-C P$ treatments. These results are in agreement with $I-V$ measurements as the increase in dark current corresponds to a decrease in the signal to noise ratio. The large photo peak shown here is associated with the $31-35 \mathrm{KeV}$ emission line of ${ }^{133} \mathrm{Ba}$. From this data it is clear that lower background noise in the detector is obtained after the $L E P-3$ treatment. Additional polishing of the edges results in increased dark current flowing between anode and cathode, which increases the RMS of the electronic noise.

For comparison commercially available spectrometer grade CZT characterized under similar laboratory conditions exhibited FWHM values ranging from $4-10 \%$ for the $31-35 \mathrm{keV}$ photo peak of $133 \mathrm{Ba}$.

1) Temporal Stability of LEP-3 Treatment: All of the detectors whose lateral edges had been polished using $3 \mu \mathrm{m} \mathrm{Al}{ }_{2} \mathrm{O}_{3}$ 


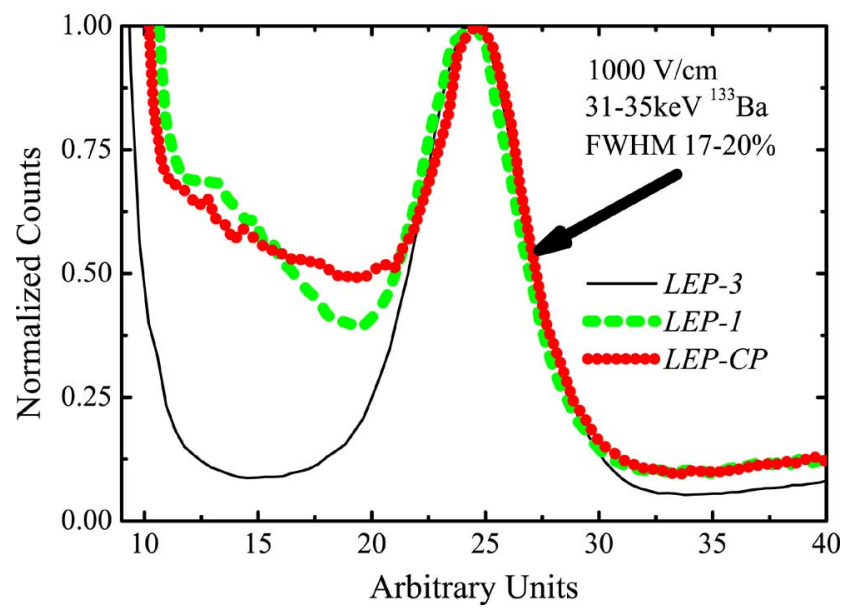

Fig. 8. Gamma response of CZT detector to ${ }^{133} \mathrm{Ba}$ after $L E P-3, L E P-1$, and $L E P-C P, \mathrm{Br}_{2}$ in ethylene glycol.

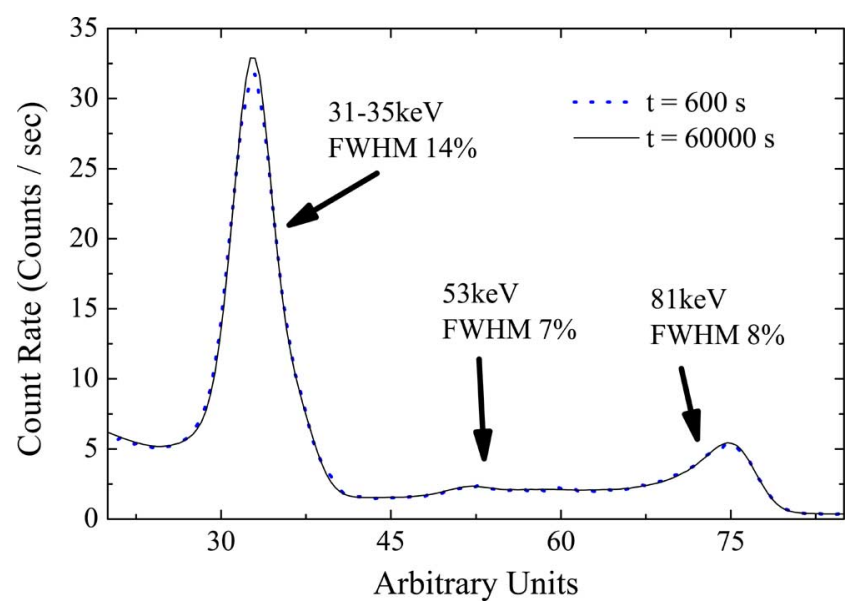

Fig. 9. Temporal gamma response of CZT detector after $L E P-3$ treatment. The $31-35 \mathrm{keV}, 53 \mathrm{keV}$, and $81 \mathrm{keV}$ peaks are all clearly visible. The detector was biased under $2000 \mathrm{~V}-\mathrm{cm}^{-1}$ for 10 hours. The detector functioned well throughout the entire measurement.

functioned at the highest voltage and did not show any signs of performance degradation during each measurement. Due to the detector failure after lapping $L E L-9$ and $L E L-3$ treatments, it was important to investigate if the detectors also had problems over longer periods of time.

To demonstrate this, the lateral edges of two CZT detectors were polished using the $L E P-3$ treatment, and the samples were biased for more than 10 hours. Presented in Figs. 9 and 10 is the gamma response for this detector after 10 minutes as well as over the 10 hour period, biased at $2500 \mathrm{~V}-\mathrm{cm}^{-1}$.

\section{DISCUSSION}

In general, improved detector performance has been observed when the lateral edges exhibit a strong luminescent band near $1.4-1.5 \mathrm{eV}$. When this luminescent emission is at its highest, more electrons in the $\mathrm{CB}$ are becoming trapped and undergoing radiative emission of photons. This radiative decay of electrons at the surface corresponds to a decrease in surface leakage current between the anode and cathode. By decreasing the concen-

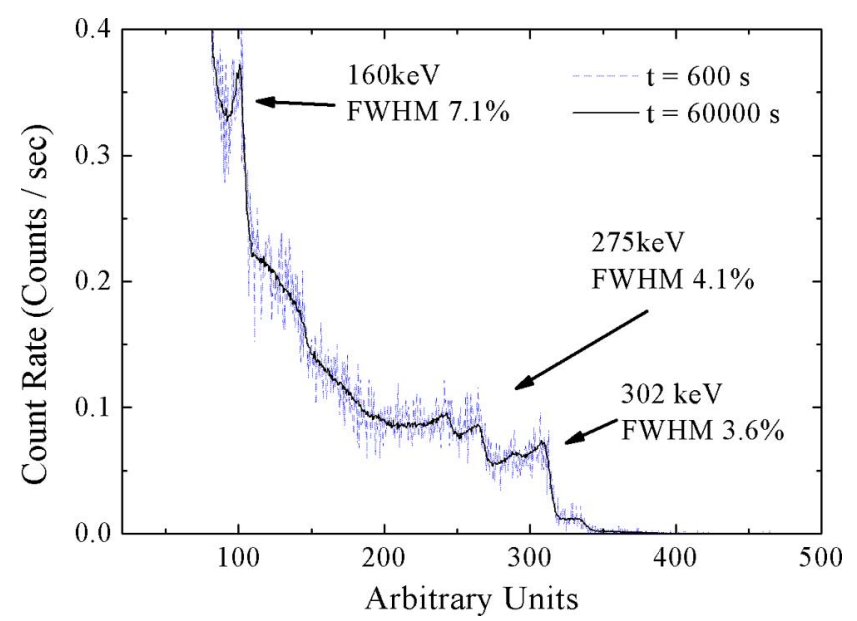

Fig. 10. Gamma response of CZT detector whose edges have been polished using $3 \mu \mathrm{m} \mathrm{Al}_{2} \mathrm{O}_{3}$, showing higher energy peaks.

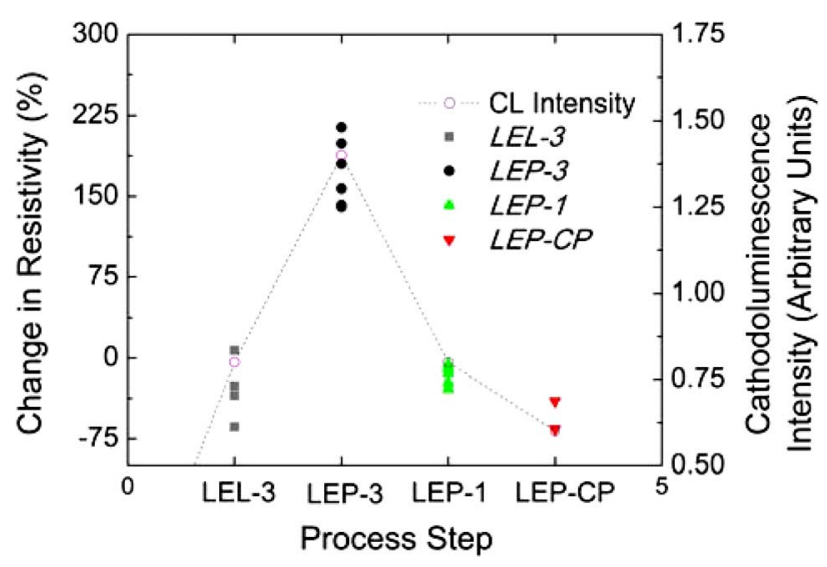

Fig. 11. Correlation between the change in resistivity and the CL emission intensity at the $1.4 \mathrm{eV}$ band.

tration of these recombination centers, less electrons can undergo this radiative recombination. As a result, dark current increases, and the noise observed in the detector increases as well.

It has been observed that this mechanism plays a strong role in the performance of planar detectors. For surfaces which exhibited luminescence, the relation between detector resistivity and the peak intensity emission in the $1.4-1.5 \mathrm{eV}$ band are presented in Fig. 11. This figure shows differences in resistivity as measured using (1) for various detectors between each subsequent processing step. Detectors were compared between two or more of the processing steps for I-V measurements to compare the effects of processing on resistivity.

From a starting point of LEL-9 edge treatment, the surface leakage current increases for each of the detectors as shown by the negative change in resistivity for LEL-3. After subsequent polishing using the LEP-3 treatment, the resistivity increases by nearly $150-200 \%$ for the detectors studied. Additional surface polishing using LEP-1 and LEP-CP reduces the resistivity by increasing bulk leakage current. This leakage current increases as a result of a lower concentration of luminescent traps on the lateral surface of CZT. These luminescent traps appear to correlate with a reduction in the surface leakage. This can be explained 
TABLE I

INCREASED LEAKAGE CURRENT AS A FUNCTION OF BIAS VOLTAGE FOR LEL AND LEP TREATMENTS COMPARED WITH LEP-3

\begin{tabular}{l|cccc} 
Treatment & \multicolumn{4}{|c}{ \% Increase in Leakage Current } \\
\hline & $500 \mathrm{~V} / \mathrm{cm}$ & $750 \mathrm{~V} / \mathrm{cm}$ & $1000 \mathrm{~V} / \mathrm{cm}$ & \# samples \\
\hline LEL-9 & $338 \%$ & $389 \%$ & $424 \%$ & 4 \\
& $\sigma=1.50$ & $\sigma=2.16$ & $\sigma=3.29$ & \\
LEL-3 & $223 \%$ & $221 \%$ & $228 \%$ & 13 \\
& $\sigma=1.49$ & $\sigma=1.56$ & $\sigma=1.82$ & \\
\hline LEP-1 & $49 \%$ & $55 \%$ & $60 \%$ & 7 \\
& $\sigma=0.57$ & $\sigma=0.46$ & $\sigma=0.49$ & \\
LEP-CP & $266 \%$ & $302 \%$ & $311 \%$ & 2 \\
& $\sigma=0.02$ & $\sigma=0.29$ & $\sigma=0.94$ &
\end{tabular}

through recombination of surface travelling electrons at the luminescent traps, where the electron recombines and a 1.4-1.5 $\mathrm{eV}$ photon is emitted.

In terms of electronic noise and improving the signal to noise ratio, the leakage current of a detector is a useful figure of merit for characterizing detector performance under operating conditions. Using the LEP-3 treatment as the treatment which provided the best performance of those treatments studied, changes in leakage current under higher bias are presented using

$$
\Delta I_{V}=\frac{I_{i}-I_{\mathrm{LEP}-3}}{I_{\mathrm{LEP}-3}}
$$

where $I_{V}$ is leakage current measured at a certain voltage (i.e., $500,750,1000), \mathrm{I}_{\mathrm{LEP}-3}$ is the leakage current measured for LEP-3 treatment, and $\mathrm{I}_{\mathrm{i}}$ represents the leakage current from the other treatments studied (i.e., LEL-9, LEL-3, LEP-1, LEP-CP).

Presented in Table I are percentage increases in leakage current, and their standard deviation for the sample set with respect to the LEP-3 treatment. The number of samples investigated using this treatment are also presented. In general, the LEL-9 and LEL-3 treatments exhibited substantially higher leakage currents, as did the LEP-CP treatment. The increasing standard deviation with increased bias was observed in all of the detectors. This is due to non-linear increases in leakage current of the test cases with respect to LEP-3, which remains constant.

Due to the thickness of the detectors and the operating voltage constraints of $+200 \mathrm{~V}$ by the Kiethley $2400 \mathrm{I}-\mathrm{V}$ system, the highest E-field obtained was between 1200 and $1000 \mathrm{~V} / \mathrm{cm}$. As can be seen in Fig. 4, non-linear increases in leakage current dominate the surface treatments LEL-9, LEL-3, LEP-CP.

\section{CONCLUSION}

Methods for improving the quality of planar detectors have been investigated. Specifically, the effect of lateral surface morphology on detector performance has been studied through the use of different lapping and polishing agents.

It has been found that polishing the edges of the planar detectors can help reduce leakage current by a factor of $200 \%$ in most cases and improve the performance of planar detectors through the introduction of surface traps. These traps provide radiative recombination centers for surface travelling electrons.

A quick 2-stage protocol is proposed which can first use a 3 $\mu \mathrm{m}$ Alumina lapping step, followed by a $3 \mu \mathrm{m}$ Alumina polishing step. Although the $L E P-1$ and $L E P-C P$ treatments provided improved surface morphology and lowered surface roughness, these results were not translated into lower leakage current at operating bias voltages. It was also been demonstrated that detectors prepared with lapped edges have a tendency to fail after period of time on the order of shorter than 1 hour and while operating at lower than optimal bias.

Through the use of CL a correlation between the 1.4-1.5 $\mathrm{eV}$ luminescent band and reduced leakage current has been observed. Using this method, results obtained from $I-V$ and Gamma ray measurements could be resolved.

\section{REFERENCES}

[1] H. Yoon et al., "Investigation of the effects of polishing and etching on the quality of Cd1-xZnxTe using spatial mapping techniques," J. Elect. Mater., vol. 26, no. 6, p. 529, 1997.

[2] Z. Zhang et al., "Chemical mechanical polishing and nanomechanics of semiconductor CdZnTe single crystals," Semicond. Sci. Technol., vol. 23, p. 105023,2008

[3] R. Singh et al., "Molecular beam epitaxy growth of high-quality $\mathrm{HgCdTe}$ LWIR layers on polished and repolished CdZnTe substrates," J. Electron. Mater., vol. 34, no. 6, pp. 885-890, 2005.

[4] P. Moravec et al., "Chemical polishing of CdZnTe substrates fabricated from crystals grown by the vertical-gradient freezing method," J. Elect. Mater., vol. 35, no. 6, pp. 1206-1213, 2006.

[5] V. G. Ivanitska et al., "Chemical etching of CdTe in aqueous solutions of H2O2-HI-citric acid," J. Electron. Mater., vol. 36, no. 8, pp. 1021-1023, 2007.

[6] Y. Cui, M. Groza, A. Burger, and R. B. James, "Effects of surface processing on the performance of Cd1-xZnxTe radiation detectors," IEEE Trans. Nucl. Sci., vol. 51, no. 3, pp. 1172-1175, Jun. 2005.

[7] M. C. Duff et al., "Effect of surface preparation technique on the radiation detector performance of CZT," Appl. Surf. Sci., vol. 254, p. 2889, 2008.

[8] A. Castaldini et al., "Cathodoluminescence and photoinduced current spectroscopy studies of defects in Cd0.8Zn 0.2Te," Phys. Rev. B, vol. 54, no. 11, p. 7622, 1996. 